\title{
Bioanalysis
}

\section{Biomarkers, PK and immunogenicity: are we ready for integration?}

\author{
"The heart of this debate, therefore, comes down to whether it is \\ necessary to have a standalone Nab assay..."
}

First draft submitted: 12 August 2016; Accepted for publication: 9 September 2016; Published online: 26 September 2016

Keywords: biomarker $\bullet$ immunogenicity $\bullet$ neutralizing antibody $\bullet$ PD $\bullet$ PK

In the context of clinical development of a therapeutic molecule, no single dataset stands on its own. As scientists we routinely integrate our datasets, fitting them together like puzzle pieces to create a more coherent picture. Examples of this include:

- Evaluating PK-PD relationships to inform therapeutic drug dosing

- Integration of PK and immunogenicity data to determine impact of immunogenicity on drug exposure

- Consideration of immunogenicity in the context of safety data

Indeed, the tiered immunogenicity testing paradigm itself involves integration of results from multiple assays - screening, confirmatory, titration and neutralizing - to develop a deeper understanding of immunogenicity and its impact.

Although the concept of data integration is not novel, there has nevertheless been ongoing robust dialogue among industry professionals and regulators regarding the integration of PK, PD and anti-drug antibody datasets to inform the presence and impact of neutralizing antibodies (Nabs). In a discussion held at the 2016 AAPS National Biotechnology Conference in Boston (MA, USA) in May of this year, this topic was distilled down to the provocative question, 'To Nab or not to Nab?' [1]. Taken at face value, this question asks whether it is necessary to measure Nab (for a given therapeutic) at all. However, the current debate has focused on a somewhat different question, which is more accurately phrased, 'To Nab assay or not to Nab assay?' Here differing views have arisen between those who believe that $\mathrm{Nab}$ assays are the gold standard and required for unequivocal Nab detection, and those who believe that an understanding of $\mathrm{Nab}$ presence and impact may also be derived from integration of other datasets (e.g., PK, PD and antidrug-antibody). The heart of this debate, therefore, comes down to whether it is necessary to have a standalone $\mathrm{Nab}$ assay or whether the integration of other datasets is also acceptable, or in some cases even preferable, for detection of $\mathrm{Nab}$ and its impact.

In our experience, some of the most hotly contested scientific debates focus on the details, where differences appear to abound, while at the heart of the matter, there is far less divergence between opposing sides. Therefore, in order to launch a truly productive discussion, it is critical to first identify common ground. To this end, we like to employ 'The Five Whys' approach. This method iteratively asks the question 'Why' to interrogate cause-and-effect relationships underlying a particular problem, with the goal of identifying the root cause. However, we have found that this approach also works well as a means to find common ground during scientific debate. So, let us apply the five 'Whys' to Nab assays:

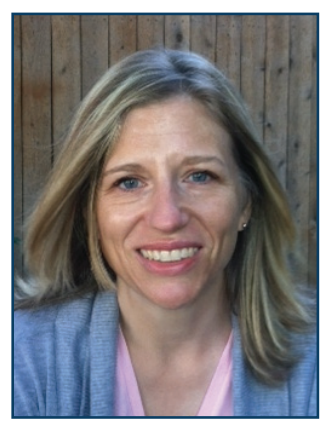

Lauren F Stevenson

Global Biomarker Discovery \& Development, Biogen,

225 Binney Street, Cambridge, MA 02142, USA

Tel.: +1 6179146479

lauren.stevenson@biogen.com 
- Why do we need a Nab assay? To enable us to detect Nabs

- Why do we need to detect Nabs? Required by regulators

- Why is it required by regulators? To understand potential impact on safety and efficacy

- Why do we need to understand potential impact on safety and efficacy? To ensure we provide safe and effective treatments to patients

- And before we even get to the fifth Why, we already have something that looks like common ground. Working from this common ground we cannot help but pose another why question: 'In order to ensure that we provide safe and effective treatments to patients, why would we not use all means at our disposal to understand the presence and impact of Nabs?'

Let us look at it from another, biomarker-oriented, perspective. We are all familiar with the use of biomarkers as indicators of other processes or responses (e.g., normal biologic and/or pathogenic processes; PD responses to a therapeutic molecule) [2]. And, although biomarkers represent an indirect tool whereby we draw conclusions about the impact of our drug through the measurement of other analytes, we are not only comfortable with this approach, but in many cases see it as necessary to fully elucidate the impact of our therapeutic treatments. So, from that perspective, is it really necessary to measure Nab 'directly' or are biomarkers of Nab equally valuable? This is the question that really lies at the heart of the ongoing debate.

A common argument in favor of standalone $\mathrm{Nab}$ assays is that with a dedicated Nab assay you 'know' you have Nab. There is some discomfort around making inferences from indirect measures in this context. Why do we trust our PD biomarkers in one scenario, but not our Nab biomarkers in another? In all cases, our comfort level should be derived from the quality of the biomarkers and the assays used to measure them. It should also be recognized that $\mathrm{Nab}$ assays themselves do not provide direct measures. Cell-based Nab assays, frequently cited as the gold standard, measure downstream functional changes (e.g., increase or decrease in an analyte downstream of a signaling pathway) [3]. They are, in fact, measuring a biomarker of the presence of $\mathrm{Nab}$ and the impact of $\mathrm{Nab}$ within the context of the assay. Indeed, many of these cell-based assays are essentially ex vivo reconstructions of PD biomarker assays, which are also able to detect the impact of $\mathrm{Nab}$, but in the context of the original biological sample. Therefore, the decision to deploy a standalone Nab assay or biomarker assay of Nab impact should occur on a case-by-case basis and take into consideration the particular therapeutic molecule, its mechanism of action, the suite of assays available to interrogate its physiological impact and the quality of those assays. The assay or assays that can best inform Nab impact should then be deployed.

This requires a change in how we think about the measurement of $\mathrm{Nabs}$, and there are genuine challenges to face, which include intellectual barriers within the scientific community as well as organizational barriers. To meet the challenge of intellectual barriers, we must commit to keeping the scientific debate open and to avoid the temptation to dichotomize and oversimplify the issue into factions of 'for $\mathrm{Nab}$ assays' or 'for integration of other assays.' We need to be in this together and 'for getting the highest quality data for the patients.' From an organizational perspective, the data integration approach will feel more intuitive in institutions where PK, biomarker and immunogenicity data generation already occur within the same department. They benefit from direct experiences where these datasets taken together have provided a sum greater than its parts. But for organizational structures where these functions are effectively separated, data integration and interpretation will be more challenging and will require a high degree of collaboration across functions. Generally speaking, change is difficult. Change in thinking, coupled with complexity in execution, even more so. But, just as degree of difficulty has never been accepted as an excuse to not develop Nab assays, neither can it be an excuse to resist change.

\section{"Why do we trust our PD biomarkers in one scenario, but not our Nab biomarkers in another?"}

So, where do we start and how can we progress together? We need to start from common ground, focus on the science and focus on the patient. To be clear, this is not a proposal by resource strapped industry to avoid the cost and effort of $\mathrm{Nab}$ assays or to abandon development of standalone $\mathrm{Nab}$ assays. This is instead a call to adopt a scientific approach, critically examine the many ways in which the question of $\mathrm{Nab}$ can be interrogated and determine the best path forward. The appropriate suite of assays that provides the best understanding of exposure, immunogenicity and its impact should be deployed. To this end, communication is essential, and we should commit to holding meaningful conversations at meetings, conferences, via online forums and through formation of focus groups. But, change will not come through conversation alone. The best means to build knowledge and drive consensus will be through the sharing of data. This should 
include mining historical data with a fresh perspective, sharing both positive and negative datasets and producing new datasets, which will together inform our understanding and enable us to determine from where the most meaningful data are derived.

In conclusion, are we ready for integration? The question is moot. In truth we already do it, but our journey is incomplete. Utilizing integrated datasets and ensuring that the best options have been explored to arrive at the best answers is the scientifically responsible approach. Starting from common ground, with open minds applying critical thinking, we must be ready to continue the journey together.

\section{References}

1 AAPS. NBC: 'To NAb or not to NAb' - what have we learned from clinical data about the utility of neutralizing antibody assessment?

https://nbc.aapsmeeting.org/event/member/231625

\section{Acknowledgements}

The author would like to thank C Stebbins and D Mehta for scientific discussions and review of the manuscript.

\section{Financial \& competing interests disclosure}

The author has no relevant affiliations or financial involvement with any organization or entity with a financial interest in or financial conflict with the subject matter for materials discussed in the manuscript. This includes employment, consultancies, honoraria, stock ownership or options, expert testimony, grants or patents received or pending, or royalties.

No writing assistance was utilized in the production of this manuscript.

2 Strimbu K, Tavel JA. What are biomarkers? Curr. Opin. HIV AIDS 5(6), 463-466 (2010).

3 Jolicoeur P, Tacey RL. Development and validation of cell-based assays for the detection of neutralizing antibodies to drug products: a practical approach. Bioanalysis 4(24), 2959-2970 (2012). 